\title{
PERIODIC SOLUTION FOR A DELAYED THREE-SPECIES FOOD-CHAIN SYSTEM WITH HOLLING TYPE-II FUNCTIONAL RESPONSE
}

\author{
QIMING LIU and RUI XU
}

Received 2 November 2002

\begin{abstract}
A delayed three-species periodic food-chain system with Holling type-II functional response is investigated. By using Gaines and Mawhin's continuation theorem of coincidence degree theory, a set of easily verifiable sufficient conditions is derived for the existence of positive periodic solutions to the system.
\end{abstract}

2000 Mathematics Subject Classification: 34K13, 92D25.

1. Introduction. A rather characteristic behavior of population dynamics is the often-observed oscillatory phenomenon of the population densities. There are three typical approaches for modelling such a behavior: (i) introducing more species into the model and considering the higher-dimensional systems (like predator-prey interactions, see May [8]); (ii) assuming that the per capita growth function is time dependent and periodic in time; (iii) taking into account the time-delay effect on the population dynamics (Smith and Kuang [9], Zhao et al. [12]). In most of the models considered so far, it has been assumed that all biological and environmental parameters are constants in time. However, any biological or environmental parameters are naturally subject to fluctuation in time. The effects of a periodically varying environment are important for evolutionary theory as the selective forces on systems in a fluctuating environment differ from those in a stable environment. Thus, the assumptions of periodicity of the parameters are a way of incorporating the periodicity of the environment (such as seasonal effects of weather, food supplies, mating habits, etc.); on the other hand, it is generally recognized that some kinds of time delays are inevitable in population interactions (see [1, 2] and the references cited therein). Time delay due to gestation is a common example because, generally, the consumption of prey by the predator throughout its past history governs the present birth rate of the predator. The effect of time delays on the asymptotic behavior of populations has been studied by a number of authors (see, e.g., [3, 11]). Therefore, more realistic models of population interactions should take into account the seasonality of the changing environment and the effect of time delays. 
Recently, Wang and Fan [10] discussed a two-species periodic predator-prey system with infinite delay. Sufficient conditions are derived in [10] for the existence of a positive periodic solution to the system. Motivated by the work of Wang and Fan in [10], in the present paper, we are devoted to the study of the following three-species periodic food-chain predator-prey system with time delays:

$$
\begin{aligned}
& \dot{x}_{1}(t)=x_{1}(t)\left[r_{1}(t)-a_{11}(t) x_{1}\left(t-\tau_{11}(t)\right)-\frac{a_{12}(t) x_{2}(t)}{1+m_{1}(t) x_{1}(t)}\right], \\
& \dot{x}_{2}(t)=x_{2}(t)\left[-r_{2}(t)+\frac{a_{21}(t) x_{1}\left(t-\tau_{21}(t)\right)}{1+m_{1}(t) x_{1}\left(t-\tau_{21}(t)\right)}-\frac{a_{23}(t) x_{3}(t)}{1+m_{2}(t) x_{2}(t)}\right], \\
& \dot{x}_{3}(t)=x_{3}(t)\left[-r_{3}(t)+\frac{a_{32}(t) x_{2}\left(t-\tau_{32}(t)\right)}{1+m_{2}(t) x_{2}\left(t-\tau_{32}(t)\right)}\right],
\end{aligned}
$$

with initial conditions

$$
x_{i}(s)=\phi_{i}(s), \quad s \in[-\tau, 0], \phi_{i}(0)>0, i=1,2,3,
$$

where $x_{1}(t), x_{2}(t)$, and $x_{3}(t)$ denote the densities of prey, predator, and top predator population, respectively. The parameter $r_{1}(t)$ is the intrinsic growth rate of the prey and $a_{11}(t)$ is the density-dependent coefficient of the prey species. The parameters $a_{12}(t)$ and $a_{23}(t)$ are the capturing rates of the predator and the top predator, respectively. The ratios $a_{21}(t) / a_{12}(t)$ and $a_{32}(t) / a_{23}(t)$ are the conversion rates of the predator and the top predator, respectively. The parameters $r_{2}(t)$ and $r_{3}(t)$ are the death rates of the predator and the top predator, respectively. The parameters $m_{1}(t)$ and $m_{2}(t)$ are the half capturing saturation rates of the predator and the top predator, respectively. The parameter $\tau_{11}(t) \geq 0$ denotes the delay due to negative feedback of the prey species $x_{1}$. The parameters $\tau_{21}(t)$ and $\tau_{32}(t)$ are the time delays due to gestation, that is, mature adult predators (top predators) can only contribute to the production of predator (top predator) biomass. The parameters $\tau_{11}(t)$, $\tau_{21}(t)$, and $\tau_{32}(t)$ are assumed to be nonnegative periodic continuous functions with common period $\omega>0, \tau=\max _{t \in[0, \omega]}\left\{\tau_{11}(t), \tau_{21}(t), \tau_{32}(t)\right\} ; a_{i j}(t)$ $(i, j=1,2,3)$ and $m_{i}(t)(i=1,2)$ are positively periodic continuous functions with period $\omega>0$.

It is well known that by the fundamental theory of functional differential equations [6], system (1.1) has a unique solution $x(t)=\left(x_{1}(t), x_{2}(t), x_{3}(t)\right)$ satisfying initial conditions (1.2). It is easy to verify that solutions of system (1.1) corresponding to initial conditions (1.2) are defined on $[0,+\infty)$ and remain positive for all $t \geq 0$. In this paper, the solution of system (1.1) satisfying initial conditions (1.2) is said to be positive.

2. Existence of periodic solutions. In this section, by using Gaines and Mawhin's continuation theorem of coincidence degree theory, we show the existence of positive $\omega$-periodic solutions of (1.1) and (1.2). To this end, we 
first introduce the following notations. Let $X, Y$ be real Banach spaces, let $L: \operatorname{Dom} L \subset X \rightarrow Y$ be a linear mapping, and let $N: X \rightarrow Y$ be a continuous mapping. The mapping $L$ is called a Fredholm mapping of index zero if $\operatorname{dim} \operatorname{Ker} L=\operatorname{codim} \operatorname{Im} L<+\infty$ and $\operatorname{Im} L$ is closed in $Y$. If $L$ is a Fredholm mapping of index zero and there exist continuous projectors $P: X \rightarrow X$ and $Q: Y \rightarrow Y$ such that $\operatorname{Im} P=\operatorname{Ker} L$ and $\operatorname{Ker} Q=\operatorname{Im} L=\operatorname{Im}(I-Q)$, then the restriction $L_{P}$ of $L$ to $\operatorname{Dom} L \cap \operatorname{Ker} P:(I-P) X \rightarrow \operatorname{Im} L$ is invertible. Denote the inverse of $L_{P}$ by $K_{P}$. If $\Omega$ is an open bounded subset of $X$, the mapping $N$ will be called $L$-compact on $\bar{\Omega}$ if $Q N(\bar{\Omega})$ is bounded and $K_{P}(I-Q) N: \bar{\Omega} \rightarrow X$ is compact. Since $\operatorname{Im} Q$ is isomorphic to $\operatorname{Ker} L$, there exists isomorphism $J: \operatorname{Im} Q \rightarrow \operatorname{Ker} L$.

For convenience of use, we introduce the continuation theorem of coincidence degree theory (see [5, page 40]) as follows.

LEMMA 2.1. Let $\Omega \subset X$ be an open bounded set. Let $L$ be a Fredholm mapping of index zero and let $N$ be $L$-compact on $\bar{\Omega}$. Assume

(a) for each $\lambda \in(0,1), x \in \partial \Omega \cap \operatorname{Dom} L, L x \neq \lambda N x$;

(b) for each $x \in \partial \Omega \cap \operatorname{Ker} L, Q N x \neq 0$;

(c) $\operatorname{deg}\{J Q N, \Omega \cap \operatorname{Ker} L, 0\} \neq 0$.

Then $L x=N x$ has at least one solution in $\bar{\Omega} \cap \operatorname{Dom} L$.

In what follows, we will use the notations

$$
\bar{f}=\frac{1}{\omega} \int_{0}^{\omega} f(t) d t, \quad f^{L}=\min _{t \in[0, \omega]} f(t), \quad f^{M}=\max _{[0, \omega]} f(t),
$$

where $f$ is a continuous $\omega$-periodic function.

LEMMA 2.2. Assume the following hold:

(H1) $\overline{a_{32}}-\overline{r_{3}} m_{2}^{M}>0$,

(H2) $\overline{r_{1}}\left(\overline{a_{21}}-m_{1}^{M} \overline{r_{2}}\right)-\overline{a_{11}} \overline{r_{2}}>0$.

Then the system of algebraic equations

$$
\begin{gathered}
\overline{r_{1}}-\overline{a_{11}} u_{1}=0 \\
-\overline{r_{2}}+\frac{1}{\omega} \int_{0}^{\omega} \frac{a_{21}(t) u_{1}}{1+m_{1}(t) u_{1}} d t-\frac{1}{\omega} \int_{0}^{\omega} \frac{a_{23}(t) u_{3}}{1+m_{2}(t) u_{2}} d t=0 \\
-\overline{r_{3}}+\frac{1}{\omega} \int_{0}^{\omega} \frac{a_{32}(t) u_{2}}{1+m_{2}(t) u_{2}} d t=0
\end{gathered}
$$

has a unique solution $\left(u_{1}^{*}, u_{2}^{*}, u_{3}^{*}\right)$ and $u_{i}^{*}>0, i=1,2,3$.

Proof. Let

$$
f\left(u_{2}\right)=-\overline{r_{3}}+\frac{1}{\omega} \int_{0}^{\omega} \frac{a_{32}(t) u_{2}}{1+m_{2}(t) u_{2}} d t .
$$


A direct calculation shows that

$$
\begin{aligned}
f^{\prime}\left(u_{2}\right) & =\frac{1}{\omega} \int_{0}^{\omega} \frac{a_{32}(t)}{\left(1+m_{2}(t) u_{2}\right)^{2}} d t>0, \quad f(0)=-\overline{r_{3}}<0, \\
\lim _{u_{2} \rightarrow+\infty} f\left(u_{2}\right) & =-\overline{r_{3}}+\overline{\left(\frac{a_{32}}{m_{2}}\right)}>-\left(\overline{r_{3}}-\frac{\overline{a_{32}}}{m_{2}^{M}}\right)>0 .
\end{aligned}
$$

Obviously, there exists a unique zero point $u_{2}^{*}>0$ such that $f\left(u_{2}^{*}\right)=0$.

The first equation of system (2.2) has a unique zero point $u_{1}^{*}=\overline{r_{1}} / \overline{a_{11}}>0$. Furthermore, from the second equation of (2.2), we obtain

$$
\begin{aligned}
\frac{u_{3}^{*}}{\omega} \int_{0}^{\omega} \frac{a_{23}(t)}{1+m_{1}(t) u_{2}^{*}} d t & =\frac{1}{\omega} \int_{0}^{\omega} \frac{a_{21}(t) u_{1}^{*}}{1+m_{1}(t) u_{1}^{*}} d t-\overline{r_{2}} \\
& \geq \frac{1}{\omega} \int_{0}^{\omega} \frac{a_{21}(t) \overline{r_{1}} / \overline{a_{11}}}{1+m_{1}^{M} \overline{r_{1}} / \overline{a_{11}}} d t-\overline{r_{2}} \\
& =\frac{\overline{r_{1}}\left(\overline{a_{21}}-m_{1}^{M} \overline{r_{2}}\right)-\overline{a_{11}} \overline{r_{2}}}{\overline{a_{11}}+m_{1}^{M} \overline{r_{1}}}>0,
\end{aligned}
$$

which yields $u_{3}^{*}>0$. The proof is complete.

We are now in a position to state our main result on the existence of a positive periodic solution to system (1.1).

THEOREM 2.3. In addition to (H1) and (H2), further assume that

(H3) $\overline{r_{1}}>\overline{r_{2}} \overline{a_{11}} /\left(\overline{a_{21}}-\overline{r_{2}} m_{1}^{M}\right) e^{2 \overline{r_{1}} \omega}+\overline{r_{3}} \overline{a_{12}} /\left(\overline{a_{32}}-m_{2}^{M} \overline{r_{3}}\right) e^{\left.2 \overline{\left(a_{21} / m_{1}\right.}\right) \omega}$.

Then system (1.1), with initial conditions (1.2), has at least one strictly positive w-periodic solution.

Proof. Since solutions of (1.1) and (1.2) remain positive for all $t \geq 0$, we let

$$
y_{1}(t)=\ln \left[x_{1}(t)\right], \quad y_{2}(t)=\ln \left[x_{2}(t)\right], \quad y_{3}(t)=\ln \left[x_{3}(t)\right]
$$

On substituting (2.6) into system (1.1), we derive

$$
\begin{aligned}
& \dot{y}_{1}(t)=r_{1}(t)-a_{11}(t) e^{y_{1}\left(t-\tau_{11}(t)\right)}-\frac{a_{12}(t) e^{y_{2}(t)}}{1+m_{1}(t) e^{y_{1}(t)}}, \\
& \dot{y}_{2}(t)=-r_{2}(t)+\frac{a_{21}(t) e^{y_{1}\left(t-\tau_{21}(t)\right)}}{1+m_{1}(t) e^{y_{1}\left(t-\tau_{21}(t)\right)}}-\frac{a_{23}(t) e^{y_{3}(t)}}{1+m_{2}(t) e^{y_{2}(t)}}, \\
& \dot{y}_{3}(t)=-r_{3}(t)+\frac{a_{32}(t) e^{y_{2}\left(t-\tau_{32}(t)\right)}}{1+m_{2}(t) e^{y_{2}\left(t-\tau_{32}(t)\right)}}
\end{aligned}
$$

It is easy to see that if system (2.7) has one $\omega$-periodic solution $\left(y_{1}^{*}(t), y_{2}^{*}(t)\right.$, $\left.y_{3}^{*}(t)\right)^{T}$, then

$$
x^{*}(t)=\left(x_{1}^{*}(t), x_{2}^{*}(t), x_{3}^{*}(t)\right)^{T}=\left(\exp \left[y_{1}^{*}(t)\right], \exp \left[y_{2}^{*}(t)\right], \exp \left[y_{3}^{*}(t)\right]\right)^{T}
$$


is a positive $\omega$-periodic solution of system (1.1). Therefore, to complete the proof, it suffices to show that system (2.7) has one $\omega$-periodic solution.

Take

$$
\begin{gathered}
X=Y=\left\{\left(y_{1}(t), y_{2}(t), y_{3}(t)\right)^{T} \in C\left(\mathbb{R}, \mathbb{R}^{3}\right): y_{i}(t+\omega)=y_{i}(t), i=1,2,3\right\}, \\
\left\|\left(y_{1}(t), y_{2}(t), y_{3}(t)\right)^{T}\right\|=\sum_{i=1}^{3} \max _{t \in[0, \omega]}\left|y_{i}(t)\right|,
\end{gathered}
$$

where $|\cdot|$ denotes the Euclidean norm. It is easy to verify that $X$ and $Y$ are Banach spaces with the norm $\|\cdot\|$. Set

$$
\begin{aligned}
& L\left(y_{1}(t), y_{2}(t), y_{3}(t)\right)^{T} \\
& \quad=\left(\frac{d y_{1}(t)}{d t}, \frac{d y_{2}(t)}{d t}, \frac{d y_{3}(t)}{d t}\right)^{T}, \quad\left(y_{1}(t), y_{2}(t), y_{3}(t)\right)^{T} \in X,
\end{aligned}
$$

and $N: X \rightarrow X$,

$$
N\left(\begin{array}{l}
y_{1}(t) \\
y_{2}(t) \\
y_{3}(t)
\end{array}\right)=\left(\begin{array}{c}
r_{1}(t)-a_{11}(t) e^{y_{1}\left(t-\tau_{11}(t)\right)}-\frac{a_{12}(t) e^{y_{2}(t)}}{1+m_{1}(t) e^{y_{1}(t)}} \\
-r_{2}(t)+\frac{a_{21}(t) e^{y_{1}\left(t-\tau_{21}(t)\right)}}{1+m_{1}(t) e^{y_{1}\left(t-\tau_{21}(t)\right)}}-\frac{a_{23}(t) e^{y_{3}(t)}}{1+m_{2}(t) e^{y_{2}(t)}} \\
-r_{3}(t)+\frac{a_{32}(t) e^{y_{2}\left(t-\tau_{32}(t)\right)}}{1+m_{2}(t) e^{y_{2}\left(t-\tau_{32}(t)\right)}}
\end{array}\right)
$$

Define two projectors $P$ and $Q$ as

$$
P\left(\begin{array}{l}
y_{1} \\
y_{2} \\
y_{3}
\end{array}\right)=Q\left(\begin{array}{l}
y_{1} \\
y_{2} \\
y_{3}
\end{array}\right)=\left(\begin{array}{l}
\frac{1}{\omega} \int_{0}^{\omega} y_{1}(t) d t \\
\frac{1}{\omega} \int_{0}^{\omega} y_{2}(t) d t \\
\frac{1}{\omega} \int_{0}^{\omega} y_{3}(t) d t
\end{array}\right), \quad\left(\begin{array}{l}
y_{1} \\
y_{2} \\
y_{3}
\end{array}\right) \in X
$$

It is clear that

$$
\begin{gathered}
\operatorname{Ker} L=\left\{x \mid x \in X, x=h, h \in \mathbb{R}^{3}\right\}, \\
\operatorname{Im} L=\left\{y \mid y \in Y, \int_{0}^{\omega} y(t) d t=0\right\} \text { is closed in } Y, \\
\operatorname{dim} \operatorname{Ker} L=\operatorname{codim} \operatorname{Im} L=3 .
\end{gathered}
$$

Therefore, $L$ is a Fredholm mapping of index zero. It is easy to show that $P$ and $Q$ are continuous projectors such that

$$
\operatorname{Im} P=\operatorname{Ker} L, \quad \operatorname{Ker} Q=\operatorname{Im} L=\operatorname{Im}(I-Q) .
$$


Furthermore, the inverse $K_{P}$ of $L_{P}$ exists, that is, $K_{P}: \operatorname{Im} L \rightarrow \operatorname{Dom} L \cap \operatorname{Ker} P$, which is given by

$$
K_{P}(y)=\int_{0}^{t} y(s) d s-\frac{1}{\omega} \int_{0}^{\omega} \int_{0}^{t} y(s) d s d t
$$

Then $Q N: X \rightarrow Y$ and $K_{P}(I-Q) N: X \rightarrow X$ read

$$
\begin{gathered}
Q N x=\left[\begin{array}{c}
\frac{1}{\omega} \int_{0}^{\omega}\left[r_{1}(t)-a_{11}(t) e^{y_{1}\left(t-\tau_{11}(t)\right)}-\frac{a_{12}(t) e^{y_{2}(t)}}{1+m_{1}(t) e^{y_{1}(t)}}\right] d t \\
\frac{1}{\omega} \int_{0}^{\omega}\left[-r_{2}(t)+\frac{a_{21}(t) e^{y_{1}\left(t-\tau_{21}(t)\right)}}{1+m_{1}(t) e^{y_{1}\left(t-\tau_{21}(t)\right)}}-\frac{a_{23}(t) e^{y_{3}(t)}}{1+m_{2}(t) e^{y_{2}(t)}}\right] d t \\
\frac{1}{\omega} \int_{0}^{\omega}\left[-r_{3}(t)+\frac{a_{32}(t) e^{y_{2}\left(t-\tau_{32}(t)\right)}}{1+m_{2}(t) e^{y_{2}\left(t-\tau_{32}(t)\right)}}\right] d t
\end{array}\right], \\
K_{P}(I-Q) N x=\int_{0}^{t} N x(s) d s-\frac{1}{\omega} \int_{0}^{\omega} \int_{0}^{t} N x(s) d s d t-\left(\frac{t}{\omega}-\frac{1}{2}\right) \int_{0}^{\omega} N x(s) d s .
\end{gathered}
$$

Clearly, $Q N$ and $K_{P}(I-Q) N$ are continuous.

In order to apply Lemma 2.1, we need to search for an appropriate open bounded subset $\Omega$.

Corresponding to the operator equation $L x=\lambda N x, \lambda \in(0,1)$, we obtain

$$
\begin{aligned}
& \dot{y}_{1}(t)=\lambda\left[r_{1}(t)-a_{11}(t) e^{y_{1}\left(t-\tau_{11}(t)\right)}-\frac{a_{12}(t) e^{y_{2}(t)}}{1+m_{1}(t) e^{y_{1}(t)}}\right], \\
& \dot{y}_{2}(t)=\lambda\left[-r_{2}(t)+\frac{a_{21}(t) e^{y_{1}\left(t-\tau_{21}(t)\right)}}{1+m_{1}(t) e^{y_{1}\left(t-\tau_{21}(t)\right)}}-\frac{a_{23}(t) e^{y_{3}(t)}}{1+m_{2}(t) e^{y_{2}(t)}}\right], \\
& \dot{y}_{3}(t)=\lambda\left[-r_{3}(t)+\frac{a_{32}(t) e^{y_{2}\left(t-\tau_{32}(t)\right)}}{1+m_{2}(t) e^{y_{2}\left(t-\tau_{32}(t)\right)}}\right] .
\end{aligned}
$$

Suppose that $\left(y_{1}(t), y_{2}(t), y_{3}(t)\right)^{T} \in X$ is a solution of system (2.17) for some $\lambda \in(0,1)$. Integrating system (2.17) over $[0, \omega]$, we have

$$
\begin{gathered}
\int_{0}^{\omega} a_{11}(t) e^{y_{1}\left(t-\tau_{11}(t)\right)} d t+\int_{0}^{\omega} \frac{a_{12}(t) e^{y_{2}(t)}}{1+m_{1}(t) e^{y_{1}(t)}} d t=\int_{0}^{\omega} r_{1}(t) d t, \\
\int_{0}^{\omega} r_{2}(t) d t+\int_{0}^{\omega} \frac{a_{23}(t) e^{y_{3}(t)}}{1+m_{2}(t) e^{y_{2}(t)}} d t=\int_{0}^{\omega} \frac{a_{21}(t) e^{y_{1}\left(t-\tau_{21}(t)\right)}}{1+m_{1}(t) e^{y_{1}\left(t-\tau_{21}(t)\right)}} d t, \\
\int_{0}^{\omega} r_{3}(t) d t=\int_{0}^{\omega} \frac{a_{32}(t) e^{y_{2}\left(t-\tau_{32}(t)\right)}}{1+m_{2}(t) e^{y_{2}\left(t-\tau_{32}(t)\right)}} d t .
\end{gathered}
$$


It follows from (2.18), (2.19), and (2.20) that

$$
\begin{aligned}
\int_{0}^{\omega} & \left|\dot{y}_{1}(t)\right| d t \\
& =\lambda \int_{0}^{\omega}\left|r_{1}(t)-a_{11}(t) e^{y_{1}\left(t-\tau_{11}(t)\right)}-\frac{a_{12}(t) e^{y_{2}(t)}}{1+m_{1}(t) e^{y_{1}(t)}}\right| d t \\
& \leq \int_{0}^{\omega}\left[r_{1}(t)+a_{11}(t) e^{y_{1}\left(t-\tau_{11}(t)\right)}+\frac{a_{12}(t) e^{y_{2}(t)}}{1+m_{1}(t) e^{y_{1}(t)}}\right] d t \\
& =2 \overline{r_{1}} \omega \triangleq d_{1}, \\
\int_{0}^{\omega} \mid & \dot{y}_{2}(t) \mid d t \\
\quad & \lambda \int_{0}^{\omega}\left|-r_{2}(t)+\frac{a_{21}(t) e^{y_{1}\left(t-\tau_{21}(t)\right)}}{1+m_{1}(t) e^{y_{1}\left(t-\tau_{21}(t)\right)}}-\frac{a_{23}(t) e^{y_{3}(t)}}{1+m_{2}(t) e^{y_{2}(t)}}\right| d t \\
& \leq \int_{0}^{\omega}\left[r_{2}(t)+\frac{a_{21}(t) e^{y_{1}\left(t-\tau_{21}(t)\right)}}{1+m_{1}(t) e^{y_{1}\left(t-\tau_{21}(t)\right)}}+\frac{a_{23}(t) e^{y_{3}(t)}}{1+m_{2}(t) e^{y_{2}(t)}}\right] d t \\
& \leq 2\left(\frac{a_{21}}{m_{1}}\right) \omega \triangleq d_{2}, \\
\int_{0}^{\omega} \mid & \dot{y}_{3}(t) \mid d t \\
& =\lambda \int_{0}^{\omega}\left|-r_{3}(t)+\frac{a_{32}(t) e^{y_{2}\left(t-\tau_{32}(t)\right)}}{1+m_{2}(t) e^{y_{2}\left(t-\tau_{32}(t)\right)}}\right| d t \\
& \leq \int_{0}^{\omega}\left[r_{3}(t)+\frac{a_{32}(t) e^{y_{2}\left(t-\tau_{32}(t)\right)}}{1+m_{2}(t) e^{y_{2}\left(t-\tau_{32}(t)\right)}}\right] d t=2 \overline{r_{3}} \omega \triangleq d_{3} .
\end{aligned}
$$

Since $\left(y_{1}(t), y_{2}(t), y_{3}(t)\right)^{T} \in X$, there exists $t_{i}, T_{i}$ such that

$$
y_{i}\left(t_{i}\right)=\min _{t \in[0, \omega]} y_{i}(t), \quad y_{i}\left(T_{i}\right)=\max _{t \in[0, \omega]} y_{i}(t), \quad i=1,2,3
$$

It follows from (2.20) that

$$
\int_{0}^{\omega} r_{3}(t) d t \geq \int_{0}^{\omega} \frac{a_{32}(t) e^{y_{2}\left(t_{2}\right)}}{1+m_{2}^{M} e^{y_{2}\left(t_{2}\right)}} d t=\frac{\overline{a_{32}} \omega e^{y_{2}\left(t_{2}\right)}}{1+m_{2}^{M} e^{y_{2}\left(t_{2}\right)}},
$$

which yields

$$
y_{2}\left(t_{2}\right) \leq \ln \frac{\overline{r_{3}}}{\overline{a_{32}}-m_{2}^{M} \overline{r_{3}}} \triangleq \rho_{2}
$$

This, together with (2.22), leads to

$$
y_{2}(t) \leq y_{2}\left(t_{2}\right)+\int_{0}^{\omega}\left|\dot{y}_{2}(t)\right| d t \leq \ln \frac{\overline{r_{3}}}{\overline{a_{32}}-m_{2}^{M} \overline{r_{3}}}+2 \overline{\left(\frac{a_{21}}{m_{1}}\right)} \omega .
$$


By (2.20) we derive

$$
\int_{0}^{\omega} r_{3}(t) d t \leq \int_{0}^{\omega} \frac{a_{32}(t) e^{y_{2}\left(T_{2}\right)}}{1+m_{2}^{L} e^{y_{2}\left(T_{2}\right)}} d t=\frac{\overline{a_{32}} \omega e^{y_{2}\left(T_{2}\right)}}{1+m_{2}^{L} e^{y_{2}\left(T_{2}\right)}},
$$

which yields

$$
y_{2}\left(T_{2}\right) \geq \ln \frac{\overline{r_{3}}}{\overline{a_{32}}-m_{2}^{L} \overline{r_{3}}} \triangleq \delta_{2}
$$

This, together with (2.22), leads to

$$
y_{2}(t) \geq y_{2}\left(T_{2}\right)-\int_{0}^{\omega}\left|\dot{y}_{2}(t)\right| d t \geq \ln \frac{\overline{r_{3}}}{\overline{a_{32}}-m_{2}^{L} \overline{r_{3}}}-2 \overline{\left(\frac{a_{21}}{m_{1}}\right)} \omega .
$$

It follows from (2.18) that

$$
\int_{0}^{\omega} a_{11}(t) e^{y_{1}\left(t-\tau_{11}(t)\right)} d t \leq \int_{0}^{\omega} r_{1}(t) d t
$$

which implies

$$
y_{1}\left(t_{1}\right) \leq \ln \frac{\overline{r_{1}}}{\overline{a_{11}}} \triangleq \rho_{1} .
$$

This, together with (2.21), leads to

$$
y_{1}(t) \leq y_{1}\left(t_{1}\right)+\int_{0}^{\omega}\left|\dot{y}_{1}(t)\right| d t \leq \ln \frac{\overline{r_{1}}}{\overline{a_{11}}}+2 \overline{r_{1}} \omega .
$$

It follows from (2.18) and (2.27) that

$$
\overline{r_{1}} \leq \overline{a_{11}} e^{y_{1}\left(T_{1}\right)}+\overline{a_{12}} e^{\left\{\ln \left[\overline{r_{3}} /\left(\overline{a_{32}}-m_{2}^{M} \overline{r_{3}}\right)\right]+2 \overline{\left(a_{21} / m_{1}\right)} \omega\right\}},
$$

which yields

$$
y_{1}\left(T_{1}\right) \geq \ln \frac{\overline{r_{1}}-\left(\overline{a_{12}} \overline{r_{3}} /\left(\overline{a_{32}}-m_{2}^{M} \overline{r_{3}}\right)\right) e^{2 \overline{\left(a_{21} / m_{1}\right)} \omega}}{\overline{a_{11}}} \triangleq \delta_{1} .
$$

This, together with (2.21), leads to

$$
\begin{aligned}
y_{1}(t) & \geq y_{1}\left(T_{1}\right)-\int_{0}^{\omega}\left|\dot{y}_{1}(t)\right| d t \\
& \geq \ln \frac{\overline{r_{1}}-\left(\overline{a_{12}} \overline{r_{3}} /\left(\overline{a_{32}}-m_{2}^{M} \overline{r_{3}}\right)\right) e^{2 \overline{\left(a_{21} / m_{1}\right)} \omega}}{\overline{a_{11}}}-2 \overline{r_{1}} \omega .
\end{aligned}
$$

By (2.19) and (2.27), we derive

$$
\frac{\overline{a_{23}} e^{y_{3}\left(t_{3}\right)}}{1+\left(m_{2}^{M} \overline{r_{3}} /\left(\overline{a_{32}}-m_{2}^{M} \overline{r_{2}}\right)\right) e^{2\left(\overline{\left.a_{21} / m_{1}\right)} \omega\right.}} \leq \overline{\left(\frac{a_{21}}{m_{1}}\right)},
$$


which yields

$$
y_{3}\left(t_{3}\right) \leq \ln \frac{\overline{\left(a_{21} / m_{1}\right)}\left(1+\left(m_{2}^{M} \overline{r_{3}} /\left(\overline{a_{32}}-m_{2}^{M} \overline{r_{2}}\right)\right) e^{2 \overline{\left(a_{21} / m_{1}\right)} \omega}\right)}{\overline{a_{23}}} \triangleq \rho_{3} .
$$

It follows from (2.23) and (2.38) that

$$
\begin{aligned}
y_{3}(t) & \leq y_{3}\left(t_{3}\right)+\int_{0}^{\omega}\left|\dot{y}_{1}(t)\right| d t \\
& \leq \ln \frac{\overline{\left(a_{21} / m_{1}\right)}\left(1+\left(m_{2}^{M} \overline{r_{3}} /\left(\overline{a_{32}}-m_{2}^{M} \overline{r_{2}}\right)\right) e^{2 \overline{\left(a_{21} / m_{1}\right)} \omega}\right)}{\overline{a_{23}}}+2 \overline{r_{3}} \omega .
\end{aligned}
$$

By (2.19) and (2.36) it follows that

$$
\begin{aligned}
& \overline{r_{2}} \omega+\overline{a_{23}} \omega e^{y_{3}\left(T_{3}\right)} \\
& \geq \int_{0}^{\omega} \frac{a_{21}(t) e^{y_{1}\left(t-\tau_{21}(t)\right)}}{1+m_{1}(t) e^{y_{1}\left(t-\tau_{21}(t)\right)}} d t \\
& \geq \int_{0}^{\omega} \frac{a_{21}(t)\left[\overline{r_{1}}-\left(\overline{a_{12}} \overline{r_{3}} /\left(\overline{a_{32}}-m_{2}^{M} \overline{r_{3}}\right)\right) e^{2 \overline{\left(a_{21} / m_{1}\right)} \omega}\right] e^{-2 \overline{r_{1}} \omega}}{\overline{a_{11}}+m_{1}^{M}\left[\overline{r_{1}}-\overline{a_{12}}\left(\overline{r_{3}} /\left(\overline{a_{32}}-m_{2}^{M} \overline{r_{3}}\right)\right) e^{2 \overline{\left(a_{21} / m_{1}\right)} \omega}\right] e^{-2 \overline{r_{1}} \omega}} d t \\
& =\frac{\overline{a_{21}} \omega\left[\overline{r_{1}}-\left(\overline{a_{12}} \overline{r_{3}} /\left(\overline{a_{32}}-m_{2}^{M} \overline{r_{3}}\right)\right) e^{2 \overline{\left(a_{21} / m_{1}\right)} \omega}\right]}{\overline{a_{11}} e^{2 \overline{r_{1}} \omega}+m_{1}^{M}\left[\overline{r_{1}}-\left(\overline{a_{12}} \overline{r_{3}} /\left(\overline{a_{32}}-m_{2}^{M} \overline{r_{3}}\right)\right) e^{2 \overline{\left(a_{21} / m_{1}\right)} \omega}\right]},
\end{aligned}
$$

which implies

$$
\begin{aligned}
& y_{3}\left(T_{3}\right) \\
& \geq \ln \left[\left(( \overline { a _ { 2 1 } } - m _ { 1 } ^ { M } \overline { r _ { 2 } } ) \left(\overline{r_{1}}-\left(\frac{\overline{r_{2}} \overline{a_{11}}}{\left(\overline{a_{21}}-\overline{r_{2}} m_{1}^{M}\right)}\right) e^{2 \overline{r_{1}} \omega}\right.\right.\right. \\
& \left.\left.\quad-\left(\frac{\overline{r_{3}} \overline{a_{12}}}{\left(\overline{a_{32}}-m_{2}^{M} \overline{r_{3}}\right)}\right) e^{2 \overline{\left(a_{21} / m_{1}\right)} \omega}\right)\right) \\
& \left.\left.\quad \times\left(\overline{a_{23}}\left[\overline{a_{11}} e^{2 \overline{r_{1}} \omega}+m_{1}^{M}\left(\overline{r_{1}}-\left(\frac{\overline{a_{12}} \overline{r_{3}}}{\left(\overline{a_{32}}-m_{2}^{M} \overline{r_{3}}\right)}\right) e^{2 \overline{\left(a_{21} / m_{1}\right)} \omega}\right)\right]\right)^{-1}\right)\right] \\
& \triangleq \delta_{3} .
\end{aligned}
$$

It follows from (2.20) and (2.41) that

$$
y_{3}(t) \geq y_{3}\left(T_{3}\right)-\int_{0}^{\omega}\left|\dot{y}_{3}(t)\right| d t \geq \delta_{3}-2 \overline{r_{3}} \omega .
$$

From what has been discussed in (2.27), (2.30), (2.33), (2.36), (2.39), and (2.42), we have

$$
\max _{t \in[0, \omega]}\left|y_{i}(t)\right| \leq \max \left\{\left|\rho_{i}\right|+d_{i},\left|\delta_{i}\right|+d_{i}\right\} \triangleq B_{i}, \quad i=1,2,3
$$


Clearly, $B_{i}(i=1,2,3)$ are independent of $\lambda$. Denote $B=B_{1}+B_{2}+B_{3}+B_{0}$; here $B_{0}$ is taken sufficiently large so that each solution $\left(v_{1}^{*}, v_{2}^{*}, v_{3}^{*}\right)^{T}$ of the system of algebraic equations

$$
\begin{gathered}
\overline{r_{1}}-\overline{a_{11}} e^{v_{1}}-\frac{1}{\omega} \int_{0}^{\omega} \frac{a_{12}(t) e^{v_{2}}}{1+m_{1}(t) e^{v_{1}}} d t=0 \\
-\overline{r_{2}}+\frac{1}{\omega} \int_{0}^{\omega} \frac{a_{21}(t) e^{v_{1}}}{1+m_{1}(t) e^{v_{1}}} d t-\frac{1}{\omega} \int_{0}^{\omega} \frac{a_{23}(t) e^{v_{3}}}{1+m_{2}(t) e^{v_{2}}} d t=0, \\
-\overline{r_{3}}+\frac{1}{\omega} \int_{0}^{\omega} \frac{a_{32}(t) e^{v_{2}}}{1+m_{2}(t) e^{v_{2}}} d t=0
\end{gathered}
$$

satisfies $\left\|\left(v_{1}^{*}, v_{2}^{*}, v_{3}^{*}\right)^{T}\right\|=\left|v_{1}^{*}\right|+\left|v_{2}^{*}\right|+\left|v_{3}^{*}\right|<B$ (if system (2.44) has solutions). Now, we take $\Omega=\left\{\left(y_{1}, y_{2}, y_{3}\right)^{T} \in X:\left\|\left(y_{1}, y_{2}, y_{3}\right)^{T}\right\|<B\right\}$. Thus, condition (a) of Lemma 2.1 is satisfied. When $\left(y_{1}, y_{2}, y_{3}\right) \in \partial \Omega \cap \operatorname{Ker} L=\partial \Omega \cap \mathbb{R}^{3}$, $\left(y_{1}, y_{2}, y_{3}\right)^{T}$ is a constant vector in $\mathbb{R}^{3}$ with $\left|y_{1}\right|+\left|y_{2}\right|+\left|y_{3}\right|=B$. If system (2.42) has solutions, then

$$
Q N\left(\begin{array}{l}
y_{1} \\
y_{2} \\
y_{3}
\end{array}\right)=\left(\begin{array}{c}
\overline{r_{1}}-\overline{a_{11}} e^{y_{1}}+\frac{1}{\omega} \int_{0}^{\omega} \frac{a_{12}(t) e^{y_{2}}}{1+m_{1}(t) e^{y_{1}}} d t \\
-\overline{r_{2}}+\frac{1}{\omega} \int_{0}^{\omega} \frac{a_{21}(t) e^{y_{1}}}{1+m_{1}(t) e^{y_{1}}} d t-\frac{1}{\omega} \int_{0}^{\omega} \frac{a_{23}(t) e^{y_{3}}}{1+m_{2}(t) e^{y_{2}}} d t \\
-\overline{r_{3}}+\frac{1}{\omega} \int_{0}^{\omega} \frac{a_{32}(t) e^{y_{2}}}{1+m_{2}(t) e^{y_{2}}} d t
\end{array}\right) \neq\left(\begin{array}{l}
0 \\
0 \\
0
\end{array}\right) .
$$

If system (2.44) does not have a solution, then we can directly derive

$$
Q N\left(\begin{array}{l}
y_{1} \\
y_{2} \\
y_{3}
\end{array}\right) \neq\left(\begin{array}{l}
0 \\
0 \\
0
\end{array}\right)
$$

Thus, condition (b) in Lemma 2.1 is satisfied.

In the following, we will prove that condition (c) in Lemma 2.1 is satisfied. To this end, we define $\phi: \operatorname{Dom} L \times[0,1] \rightarrow X$ by

$$
\begin{aligned}
\phi\left(y_{1}, y_{2}, y_{3}, \mu\right)= & \left(\begin{array}{c}
\overline{r_{2}}+\frac{1}{\omega} \int_{0}^{\omega} \frac{a_{21}(t) e^{y_{1}}}{1+m_{1}(t) e^{y_{1}}} d t-\frac{1}{\omega} \int_{0}^{\omega} \frac{a_{23}(t) e^{y_{3}}}{1+m_{2}(t) e^{y_{2}}} d t \\
-\overline{r_{3}}+\frac{1}{\omega} \int_{0}^{\omega} \frac{a_{32}(t) e^{y_{2}}}{1+m_{2}(t) e^{y_{2}}} d t
\end{array}\right) \\
& +\mu\left(\begin{array}{c}
-\frac{1}{\omega} \int_{0}^{\omega} \frac{a_{12}(t) e^{y_{2}}}{1+m_{1}(t) e^{y_{1}}} d t \\
0 \\
0
\end{array}\right),
\end{aligned}
$$


where $\mu$ is a parameter. When $\left(y_{1}, y_{2}, y_{3}\right)^{T} \in \partial \Omega \cap \mathbb{R}^{3},\left(y_{1}, y_{2}, y_{3}\right)^{T}$ is a constant vector in $\mathbb{R}^{3}$ with $\left|y_{1}\right|+\left|y_{2}\right|+\left|y_{3}\right|=B$. We will show that when $\left(y_{1}, y_{2}\right.$, $\left.y_{3}\right)^{T} \in \partial \Omega \cap \operatorname{Ker} L, \phi\left(y_{1}, y_{2}, y_{3}, \mu\right) \neq 0$. If the conclusion is not true, then there is a constant vector $\left(y_{1}, y_{2}, y_{3}\right)^{T} \in \mathbb{R}^{3}$ with $\left|y_{1}\right|+\left|y_{2}\right|+\left|y_{3}\right|=M$ satisfying $\phi\left(y_{1}, y_{2}, y_{3}, \mu\right)=0$, that is,

$$
\begin{gathered}
\overline{r_{1}}-\overline{a_{11}} e^{y_{1}}-\mu \frac{1}{\omega} \int_{0}^{\omega} \frac{a_{12}(t) e^{y_{2}}}{1+m_{1}(t) e^{y_{1}}} d t=0 \\
-\overline{r_{2}}+\frac{1}{\omega} \int_{0}^{\omega} \frac{a_{21}(t) e^{y_{1}}}{1+m_{1}(t) e^{y_{1}}} d t-\frac{1}{\omega} \int_{0}^{\omega} \frac{a_{23}(t) e^{y_{3}}}{1+m_{2}(t) e^{y_{2}}} d t=0, \\
-\overline{r_{3}}+\frac{1}{\omega} \int_{0}^{\omega} \frac{a_{32}(t) e^{y_{2}}}{1+m_{2}(t) e^{y_{2}}} d t=0 .
\end{gathered}
$$

A similar argument in (2.27), (2.30), (2.33), (2.36), (2.39), and (2.42) shows that

$$
\left|y_{i}\right| \leq \max \left\{\left|\delta_{i}\right|,\left|\rho_{i}\right|\right\}, \quad i=1,2,3
$$

Thus

$$
\left|y_{1}\right|+\left|y_{2}\right|+\left|y_{3}\right| \leq \sum_{i=1}^{3} \max \left\{\left|\rho_{i}\right|,\left|\delta_{i}\right|\right\}<B
$$

which is a contradiction. Using the property of topological degree and taking $J=I: \operatorname{Im} Q \rightarrow \operatorname{Ker} L,\left(y_{1}, y_{2}, y_{3}\right)^{T} \rightarrow\left(y_{1}, y_{2}, y_{3}\right)^{T}$, we have

$$
\begin{aligned}
\operatorname{deg}\left(J Q N\left(y_{1}, y_{2}, y_{3}\right)^{T}, \Omega \cap \operatorname{Ker} L,(0,0,0)^{T}\right) \\
=\operatorname{deg}\left(\phi\left(y_{1}, y_{2}, y_{3}, 1\right), \Omega \cap \operatorname{Ker} L,(0,0,0)^{T}\right) \\
=\operatorname{deg}\left(\phi\left(y_{1}, y_{2}, y_{3}, 0\right), \Omega \cap \operatorname{Ker} L,(0,0,0)^{T}\right) \\
=\operatorname{deg}\left(\left(\overline{r_{1}}-\overline{a_{11}} e^{y_{1}},-\overline{r_{2}}+\frac{1}{\omega} \int_{0}^{\omega} \frac{a_{21}(t) e^{y_{1}}}{1+m_{1}(t) e^{y_{1}}} d t-\frac{1}{\omega} \int_{0}^{\omega} \frac{a_{23}(t) e^{y_{3}}}{1+m_{2}(t) e^{y_{2}}} d t,\right.\right. \\
\left.\left.\quad-\overline{r_{3}}+\frac{1}{\omega} \int_{0}^{\omega} \frac{a_{32}(t) e^{y_{2}}}{1+m_{2}(t) e^{y_{2}}} d t\right)^{T}, \Omega \cap \operatorname{Ker} L,(0,0,0)^{T}\right) .
\end{aligned}
$$

Under the assumptions (H1)-(H3), by Lemma 2.2 we see that system (2.2) has a unique solution $\left(u_{1}^{*}, u_{2}^{*}, u_{3}^{*}\right)^{T}$ and $u_{i}^{*}>0, i=1,2,3$. Thus, a direct and standard calculation shows that

$$
\operatorname{deg}\left(J Q N\left(y_{1}, y_{2}, y_{3}\right)^{T}, \Omega \cap \operatorname{Ker} L,(0,0,0)^{T}\right)=-1
$$

Finally, it is easy to show that the set $\left\{K_{P}(I-Q) N u \mid u \in \bar{\Omega}\right\}$ is equicontinuous and uniformly bounded. By using the Arzelá-Ascoli theorem, we see that $K_{P}(I-Q) N: \bar{\Omega} \rightarrow X$ is compact. Moreover, $Q N(\bar{\Omega})$ is bounded. Consequently, $N$ is $L$-compact. 
By now we have proved that $\Omega$ satisfies all the requirements in Lemma 2.1. Hence, system (2.7) has at least one $\omega$-periodic solution. Accordingly, system (1.1) has at least one positive $\omega$-periodic solution. This completes the proof.

3. Discussion. If all the biological and environmental parameters of system (1.1) are constants, then system (1.1) reduces to the following autonomous differential system:

$$
\begin{aligned}
& \dot{x}_{1}(t)=x_{1}(t)\left[r_{1}-a_{11} x_{1}\left(t-\tau_{11}\right)-\frac{a_{12} x_{2}(t)}{1+m_{1} x_{1}(t)}\right], \\
& \dot{x}_{2}(t)=x_{2}(t)\left[-r_{2}+\frac{a_{21} x_{1}\left(t-\tau_{21}\right)}{1+m_{1} x_{1}\left(t-\tau_{21}\right)}-\frac{a_{23} x_{3}(t)}{1+m_{2} x_{2}(t)}\right], \\
& \dot{x}_{3}(t)=x_{3}(t)\left[-r_{3}+\frac{a_{32} x_{2}\left(t-\tau_{32}\right)}{1+m_{2} x_{2}\left(t-\tau_{32}\right)}\right],
\end{aligned}
$$

where $a_{i j}, r_{i}$, and $m_{i}$ are positive constants, $\tau_{11}, \tau_{21}$, and $\tau_{32}$ are nonnegative constants.

Corresponding to Theorem 2.3, we have the following conclusion.

THEOREM 3.1. System (3.1) admits at least one positive equilibrium provided that

$(\mathrm{H} 1)^{\prime} \quad a_{32}-m_{2} r_{3}>0$,

(H2) $r_{1}\left(a_{21}-m_{1} r_{2}\right)-a_{11} r_{2}>0$,

(H3)' $r_{1}>r_{2} a_{11} /\left(a_{21}-r_{2} m_{1}\right)+r_{3} a_{12} /\left(a_{32}-m_{2} r_{3}\right)$.

Proof. Consider the following system of algebraic equations:

$$
\begin{aligned}
r_{1}-a_{11} x_{1}^{*}-\frac{a_{12} x_{2}^{*}}{1+m_{1} x_{1}^{*}} & =0, \quad-r_{2}+\frac{a_{21} x_{1}^{*}}{1+m_{1} x_{1}^{*}}-\frac{a_{23} x_{3}^{*}}{1+m_{2} x_{2}^{*}}=0, \\
-r_{3}+\frac{a_{32} x_{2}^{*}}{1+m_{2} x_{2}^{*}}=0 . &
\end{aligned}
$$

The third equation of system (3.2) has a unique zero point $x_{2}^{*}=r_{3} /\left(a_{32}-\right.$ $\left.m_{2} r_{3}\right) \triangleq A$. On substituting $x_{2}^{*}=A$ into the first equation of (3.2), we obtain

$$
r_{1}-a_{11} x_{1}^{*}-\frac{a_{12} A}{1+m_{1} x_{1}^{*}}=0
$$

that is

$$
a_{11} m_{1} x_{1}^{*^{2}}+\left(a_{11}-m_{1} r_{1}\right) x_{1}^{*}-\left(r_{1}-a_{12} A\right)=0
$$

Let $\Delta=\left(a_{11}-m_{1} r_{1}\right)^{2}+4 a_{11} m_{1}\left(r_{1}-a_{12} A\right)$. It follows from (H3)' that $r_{1}-$ $a_{12} A>0$. Thus we see that (3.4) has a unique positive solution $x_{1}^{*}$. It follows from the first equation of system (3.2) that $x_{1}^{*}>\left(r_{1}-a_{12} A\right) / a_{11}$. Therefore, 
from the third equation of system (3.4), we obtain

$$
\begin{aligned}
\frac{a_{23} x_{3}^{*}}{1+m_{2} x_{2}^{*}} & =\frac{a_{21} x_{1}^{*}}{1+m_{1} x_{1}^{*}}-r_{2} \\
& >\frac{a_{21}\left(\left(r_{1}-a_{12} A\right) / a_{11}\right)}{1+m_{1}\left(\left(r_{1}-a_{12} A\right) / a_{11}\right)}-r_{2} \\
& =\frac{\left(a_{21}-m_{1} r_{2}\right)\left(r_{1}-r_{2} a_{11} /\left(a_{21}-r_{2} m_{1}\right)-r_{3} a_{12} /\left(a_{32}-m_{2} r_{3}\right)\right)}{a_{11}+m_{1}\left(r_{1}-a_{12} r_{3} /\left(a_{32}-m_{2} r_{3}\right)\right)}>0 .
\end{aligned}
$$

Hence it follows that $x_{3}^{*}>0$. This completes the proof.

In this paper, we have combined the effects of periodicity of the environment and time delays on the dynamics of a food-chain model with Holling type-II functional response. By using Gaines and Mawhin's continuation theorem of coincidence degree theory, we have discussed the existence of positive periodic solutions of the model.

We note that assumptions (H1), (H2), and (H3) in Theorem 2.3 are equivalent to the following:

(i) $\overline{a_{32}}>\overline{r_{3}} m_{2}^{M}$,

(ii) $\overline{a_{21}}>\overline{r_{2}} m_{1}^{M}$,

(iii) $\overline{r_{1}}-\left(\overline{r_{2}} \overline{a_{11}} /\left(\overline{a_{21}}-\overline{r_{2}} m_{1}^{M}\right)\right) e^{2 \overline{r_{1}} \omega}>\left(\overline{r_{3}} \overline{a_{12}} /\left(\overline{a_{32}}-m_{2}^{M} \overline{r_{3}}\right)\right) e^{2 \overline{\left(a_{21} / m_{1}\right)} \omega}$.

By Theorem 2.3, we see that system (1.1), with initial conditions (1.2), will have at least one periodic solution if the intrinsic growth rate of the prey species and the conversion rates of the predator and the top predator are high, and the density-dependent coefficient of the prey, the death rate of the predator and the top predator are low enough. By Theorem 2.3, we see that the time delays are harmless to the existence of positive periodic solutions.

An alternative method in proving the existence of positive periodic solutions of system (1.1) may be the application of Horn's asymptotic fixed-point theorem (see, e.g., [4, 7]), while this method allows the investigator to address the stability issue of the periodic solutions. This may be our future work.

We would like to mention here that it is interesting but challenging to discuss the global attractiveness of positive periodic solutions of system (1.1) when all its coefficients are periodic functions with a common period. We leave this for our future work.

Acknowledgment. The authors are very grateful to the referee for careful reading of the manuscript and many valuable comments and suggestions, which greatly improved the presentation of this work.

\section{REFERENCES}

[1] J. M. Cushing, Integrodifferential Equations and Delay Models in Population Dynamics, Lecture Notes in Biomathematics, vol. 20, Springer-Verlag, New York, 1977. 
[2] H. I. Freedman and V. S. H. Rao, The trade-off between mutual interference and time lags in predator-prey systems, Bull. Math. Biol. 45 (1983), no. 6, 991-1004.

[3] H. I. Freedman, J. W.-H. So, and P. Waltman, Coexistence in a model of competition in the chemostat incorporating discrete delays, SIAM J. Appl. Math. 49 (1989), no. 3, 859-870.

[4] H. I. Freedman and J. H. Wu, Periodic solutions of single-species models with periodic delay, SIAM J. Math. Anal. 23 (1992), no. 3, 689-701.

[5] R. E. Gaines and J. L. Mawhin, Coincidence Degree, and Nonlinear Differential Equations, Springer-Verlag, Berlin, 1977, 38-48.

[6] J. Hale, Theory of Functional Differential Equations, Springer-Verlag, New York, 1977.

[7] W. A. Horn, Some fixed point theorems for compact maps and flows in Banach spaces, Trans. Amer. Math. Soc. 149 (1970), 391-404.

[8] R. M. May, Stability and Complexity in Model Ecosystems, Princeton University Press, Princeton, 1974.

[9] H. L. Smith and Y. Kuang, Periodic solutions of differential delay equations with threshold-type delays, Oscillation and Dynamics in Delay Equations (San Francisco, CA, 1991) (J. Graef and J. Hale, eds.), Contemp. Math., vol. 129, American Mathematical Society, Rhode Island, 1992, pp. 153-176.

[10] K. Wang and M. Fan, Positive periodic solutions of predator-prey systems with infinite delay, Chinese Ann. Math. Ser. B 21 (2000), no. 1, 43-54.

[11] W. Wendi and M. Zhien, Harmless delays for uniform persistence, J. Math. Anal. Appl. 158 (1991), no. 1, 256-268.

[12] T. Zhao, Y. Kuang, and H. L. Smith, Global existence of periodic solutions in a class of delayed Gause-type predator-prey systems, Nonlinear Anal. 28 (1997), no. 8, 1373-1394.

Qiming Liu: Department of Mathematics, Institute of Shijiazhuang Mechanical Engineering, Shijiazhuang 050003, China

E-mail address: 11qm2002@yahoo.com.cn

Rui Xu: Department of Mathematics, Institute of Shijiazhuang Mechanical Engineering, Shijiazhuang 050003, China

Current address: Department of Mathematics, University of Dundee, Dundee DD1 $4 \mathrm{HN}$, UK

E-mail address: ruixu@maths.dundee.ac.uk 


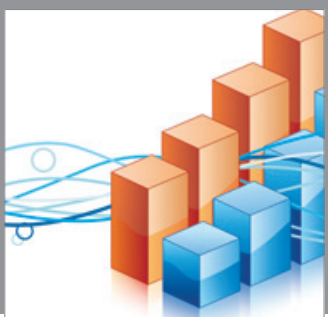

Advances in

Operations Research

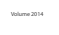

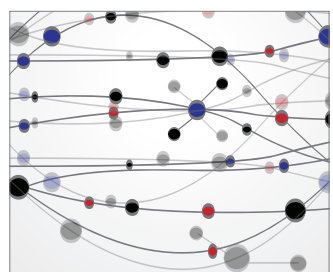

\section{The Scientific} World Journal
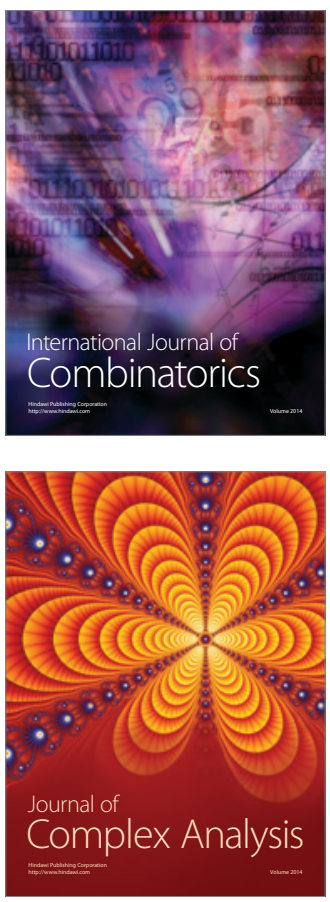

International Journal of

Mathematics and

Mathematical

Sciences
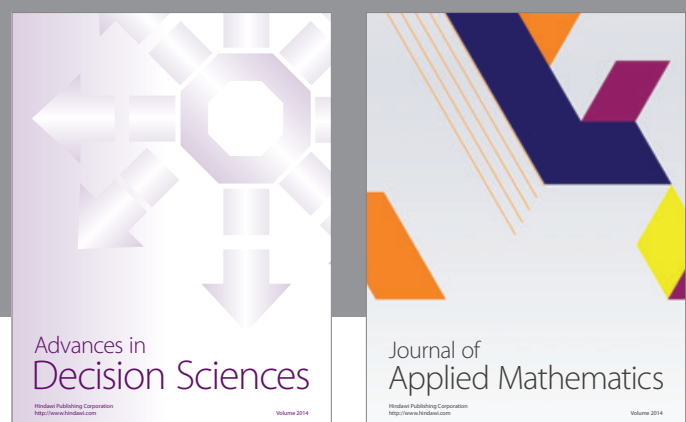

Journal of

Applied Mathematics
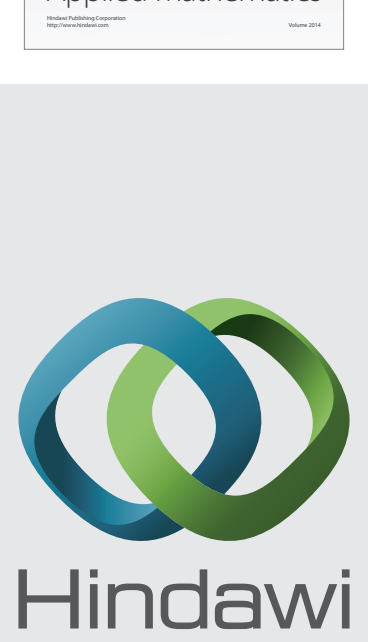

Submit your manuscripts at http://www.hindawi.com
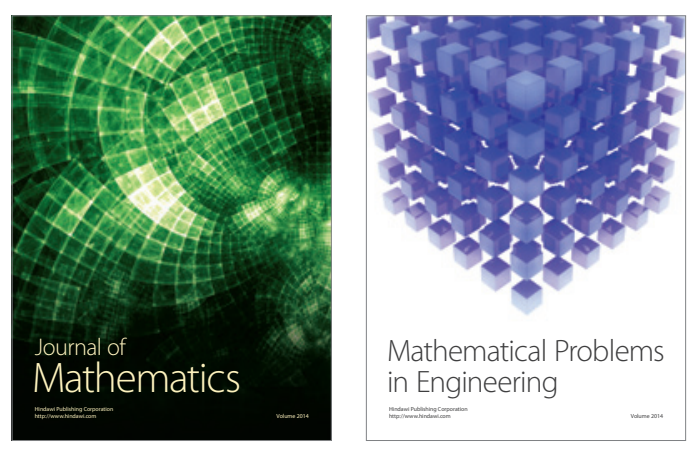

Mathematical Problems in Engineering
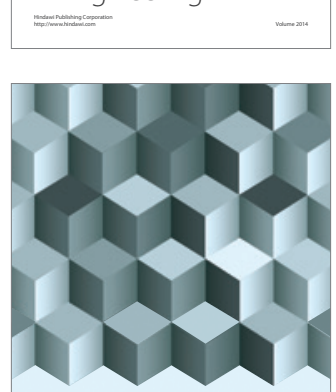

Journal of

Function Spaces
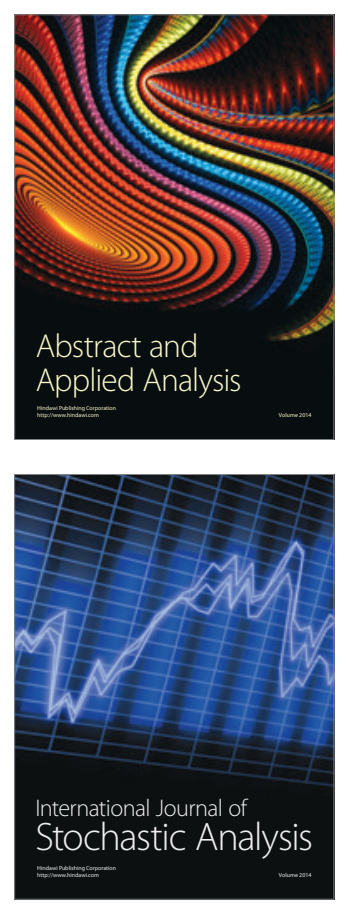

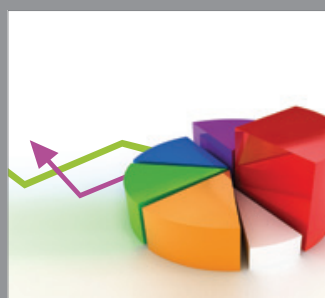

ournal of

Probability and Statistics

Promensencen
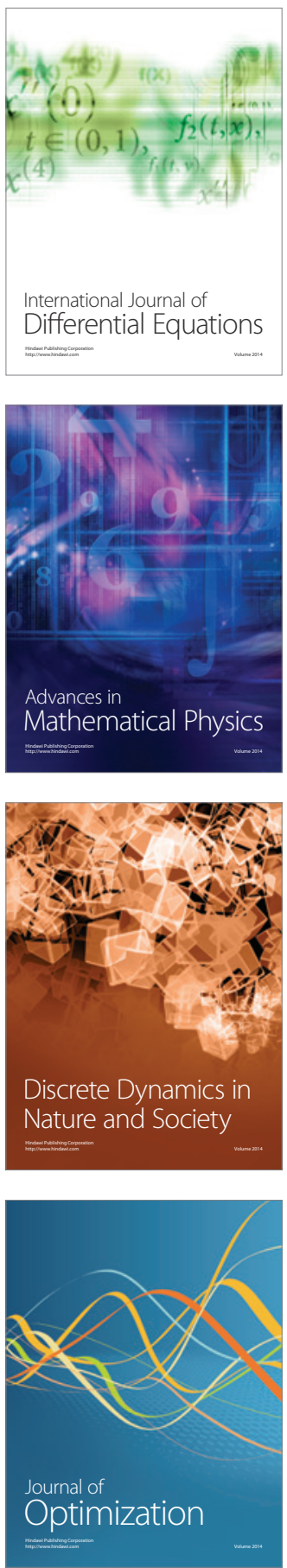